similar to that of active spiral galaxies.

One of the most spectacular results from IRAS is the discovery by Aumanet al. of an infrared excess, extending to 20 arc $s$ at 60 $\mu \mathrm{m}$, around the bright star Vega. The most likely origin of the infrared emission is thermal radiation from solid grains, at least $1 \mathrm{~mm}$ in radius - smaller grains would have spiralled into Vega by the PoyntingRobertson effect - and heated by light from Vega to about $85 \mathrm{~K}$. The grains must lie in a shell or ring at a distance of about 85 AU from Vega and in orbit around the star. These particles cannot have been present since the time of formation of Vega, some $1-2 \times 10^{8}$ yr ago, since they are much larger than typical interstellar grains (which are at most $0.5 \mu \mathrm{m}$ in size). Clearly they have grown considerably since Vega formed. Since our Solar System is about $4.5 \times 10^{9} \mathrm{yr}$ old, it is tempting to infer that the shell around Vega represents some intermediate stage in the formation of a planetary system.

Another major surprise from IRAS is the discovery of interstellar cirrus. Low et $a l$. report extended patches of far-infrared emission, seen predominantly at $60 \mu \mathrm{m}$ and $100 \mu \mathrm{m}$, both high above the galactic plane and in the ecliptic plane. Three components have been identified: one associated with neutral hydrogen and dust concentrations at very high galactic latitudes; another in the ecliptic, associated with the interplanetary medium and apparently running continuously around the Solar System; and a third - the coldest - which correlates poorly with any known structure in the Galaxy or Solar System. Degree-size patches of $100 \mu \mathrm{m}$ emission are seen and could represent either dust clouds in the outer Solar System, or a novel component of the interstellar medium. IRAS observations, taken six months apart, should be capable of resolving this enigma.

Finally, the IRAS minisurvey has revealed some very intriguing unidentified objects that may be of a fundamentally new type. Houck et al. identify nine point sources that are bright at $60 \mu \mathrm{m}$ but have no obvious identifiable optical counterpart brighter than $18.5 \mathrm{mag}$. The intrinsic infrared luminosity exceeds that in the blue band by at least an order of magnitude. Of course, these sources may not be extragalactic or even outside the Solar System. Nevertheless, their discovery is one of the potentially most important accomplishments of IRAS. Radio surveys led to the discovery of quasars and pulsars; X-ray surveys came up with $\mathrm{X}$-ray binaries and bursters; now the race will begin to explain the unidentified sources in the first all-sky infrared survey.

During the next decade both the European Space Agency and the National Aeronautics and Space Administration have plans for a sequel to IRAS. Both the European (ISO for Infrared Space Observatory) and the American (SIRTF for Shuttle Infrared Telescope Facility) experiments are designed to be operated as

true observatories for infrared astronomy, carrying a variety of focal-plane instruments. Cryogenically cooled 1-m class space telescopes will be able to study the infrared sources discovered by IRAS at more than 100 times the sensitivity and spectral resolution and provide detailed pictures of even the faintest IRAS sources. This quantum leap in infrared capability

\title{
Radiography of the living brain
}

IT was not until thirty years after the first $\mathrm{X}$-ray images of the skull that the first cerebral angiograms, obtained by Egas Moniz and shown here, revealed the distribution of tissue, blood and water in the brain.

A neurologist at Santa Maria Hospital in Lisbon, Moniz planned to inject a contrast agent in order "to make the diagnosis of the localization of the majority of [brain] tumours through the alteration of the normal arterial pattern of the cerebrum". The idea was not new: as early as $1896 \mathrm{E}$. Haschek and O.T. Lindenthal in Vienna studied the hand of a corpse through injection of Teichmann's mixture, a toxic amalgam of petroleum, quicklime and mercuric sulphide. But to perform angiography on a living patient, Moniz had to find a less hazardous contrast agent and a way to inject it so that good photographs could be taken before it was heavily diluted by the blood flow.

The method he adopted consisted of exposing the internal carotid artery, injecting the contrast agent, immediately placing a ligature on the artery and then releasing it a split second before taking the radiogram. With his third subject Moniz achieved success at an exposure of $0.25 \mathrm{~s}$ using a 25 per cent solution of sodium will do for the infrared astronomers what the Einstein Observatory did for X-ray astronomers. Only then will we see more than the tantalizing glimpse of the infrared sky that IRAS has already provided.

Joseph Silk is at the Astronomy Department, University of California, Berkeley, California 94720. chloride. "At that unforgettable hour, the afternoon of 28th of June 1927," wrote Moniz later, "all our attention was concentrated upon the first arteriogram. We remember with pleasure the work done, . . . the culmination of an obsession to realize a long-standing project. On the film [shown below] the cerebral vessels were seen, but deformed through the presence of the tumour. The internal carotid artery was pulled forward. . . The sylvian artery was displaced upward from its normal position."

Within the next decade another thousand diagnoses by means of angiography had been successfully conducted and the technique had been applied by Reynaldo dos Santos, Moniz and others to obtain arteriograms of other parts of the body. Ironically, Moniz was to share the 1949 Nobel Prize for Medicine on the strength of originating the technique of prefrontal lobotomy, a practice later discredited, whereas his lasting contribution to cerebral angiography went unrecognized at the time. Jon Darius Selected from Beyond Vision by Jon Darius of the Science Museum, London (to be published in April by Oxford University Press). Radiogram reproduced by courtesy of Antonio Coito of the Santa Maria Hospital in Lisbon, Portugal.

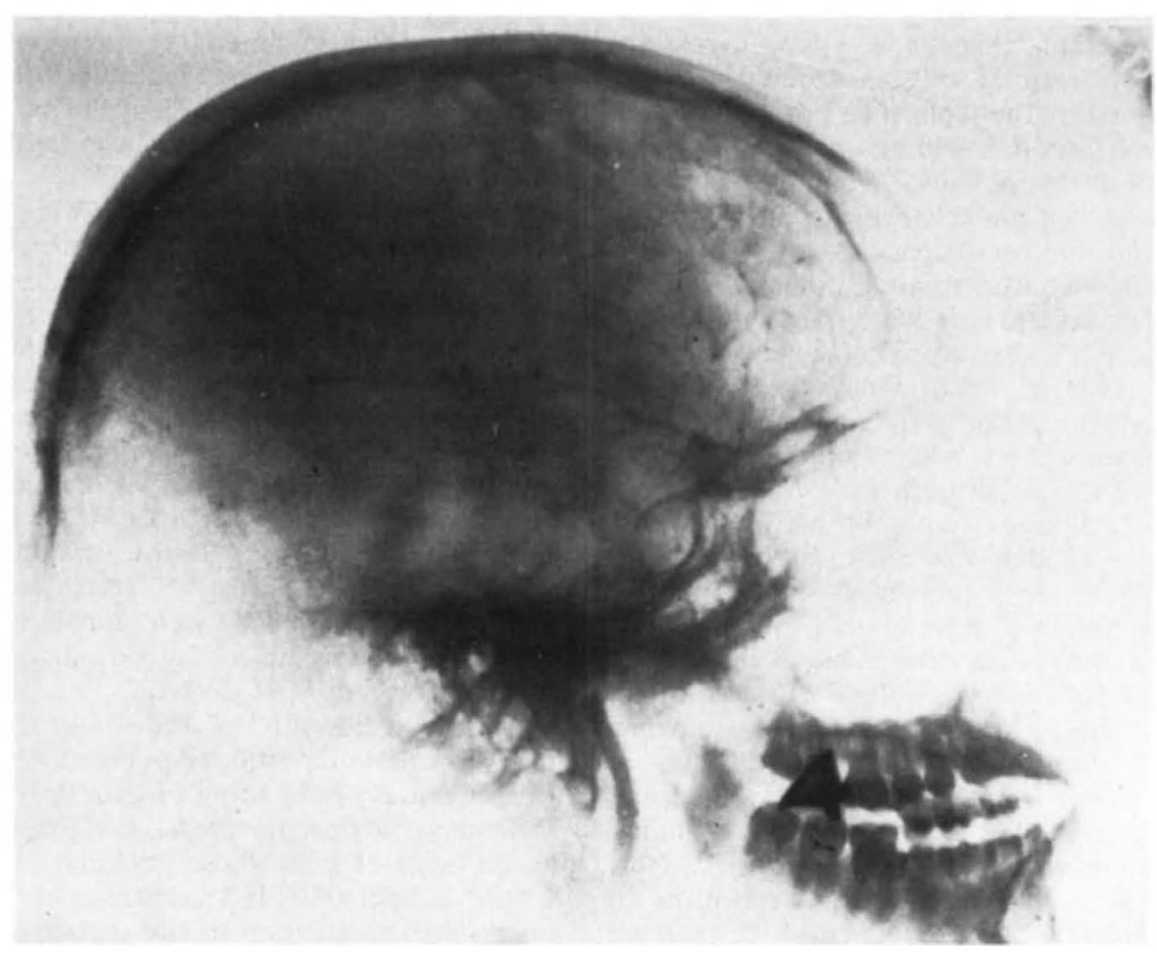

